\title{
Câncer de Mama e Colo do Útero: estrutura organizacional da prevenção na estratégia saúde da família
}

Resumo: Este artigo objetiva caracterizar a organização estrutural de unidades básicas da Estratégia Saúde da Família(ESF), com vistas à efetivação da assistência na prevenção dos cânceres de mama e de colo de útero. Estudo transversal, de abordagem quantitativa, desenvolvida em 61 unidades da ESF. Os resultados revelaram a falta de alguns recursos de infraestrutura importantes para a execução do trabalho do enfermeiro, assim como a falta de alguns profissionais. A falta de espaços apropriados para a efetivação da assistência à prevenção de ambos os cânceres interfere diretamente na qualidade da assistência prestada.

Descritores: Prevenção de Câncer de Mama, Neoplasia Uterina, Atenção Primária à Saúde

\section{Breast and cervical cancers: organizational structure of prevention in Family's Health Strategy}

Abstract: This article aims to characterize the organizational structure of the basics facilities of Family Health Strategy (FHS), aiming the effectiveness of assistance in the prevention of the breast and cervical cancers. Cross-sectional study, a quantitative approach, developed in 61 units of FHS. The results revealed the lack of some resources in the major infrastructure for the implementation of nursing work, as well as the lack of key professionals. Lack of appropriated spaces to the effective assistance prevention of both cancers affects directly the quality of the assistance. Descriptors: Breast Cancer Prevention, Uterine Neoplasia, Primary Health Care

\section{Cáncer de mama y Cáncer de cuello uterino: la prevención en la estructura organizativa de la Estrategia Salud de laFamilia.}

Resumen: Este artículo tiene como objetivo caracterizar la organización estructural de las unidades básicas de la Estrategia Salud de La Familia (ESF), con vistas a efectivación de la assistência em la prevención de los cánceres de la mama e del cuello uterino. Estudio transversal, de enfoque cuantitativo, desarollado em 61 unidades del ESF. Los resultados revelaran la falta de algunos recursos de la infraestructura importantes para la execución del trabajo de lo enfermero, así como la falta de algunos profesionales. La falta de los espacios apropriados para la efecacia de la asistencia a prevención de ambos los cánceres interfiere diretamente em la calidad prestada.

Descriptores: Prevención de Cáncer de Mama, Neoplasia Del Cuello Uterino Atención, Primaria de Salud.

\section{INTRODUÇÃO}

E sta pesquisa teve como objeto de estudo a organização estrutural de unidades de saúde da Atenção Primária à Saúde (APS) do município de Cuiabá, capital do Estado de Mato Grosso- Brasil, para a prevenção dos dois tipos de cânceres mais incidentes na população feminina brasileira, o câncer de mama e o câncer de colo do útero.

As neoplasias representaram, no início do século XXI, um problema de saúde pública em todo o mundo. Entre as mulheres, as neoplasias de mama e colo do útero são os tipos mais frequentes ${ }^{(1)}$. O câncer de mama é o segundo tipo mais frequente no mundo e o mais comum entre as mulheres, no Brasil, as taxas de mortalidade por este tipo de câncer continuam elevadas, muito provavelmente porque a doença ainda é diagnosticada em estágios avançados. O câncer do colo do útero por sua vez, é o segundo mais incidente na população feminina brasileira, excetuando-se os casos de câncer de pele não melanoma ${ }^{(2)}$.

Em relação à neoplasia mamária, não há estratégias específicas que possibilitem sua prevenção, porém, sabe-se da importância da detecção precoce para um tratamento eficaz, desta forma, a estratégia fundamental é a educação da mulher e dos profissionais de saúde para o reconhecimento dos sinais e sintomas do câncer de mama, assim como o acesso rápido e facilitado aos serviços de saúde ${ }^{(3)}$. O câncer de colo do útero, por sua vez, tem como principal característica ser passível de prevenção, podendo ser detectado antes da forma invasiva. Contudo, ainda representa um importante e permanente desafio para os gestores na área da saúde pública.

A Estratégia Saúde da Família (ESF) surgiu no Brasil, como uma tática de reorientação do modelo assistencial, operacionalizada mediante a implantação de equipes multiprofissionais em unidades básicas de saúde. As equipes atuam implementando ações de promoção da saúde, prevenção, recuperação, reabilitação de doenças e agravos mais frequentes, e na manutenção da saúde da comunidade em que atuam. Esta estratégia, por meio da atuação de equipes multiprofissionais, busca acompanhar de perto as famílias em seu contexto territorial, de forma a responder às demandas apresentadas pela clientela, mas também, desenvolver ações de vigilância à saúde ${ }^{(4)}$.

A ESF enfrenta sérios problemas que impedem prestação de

'Aluna de Gradução do curso Enfermagem da FAEN/UFMT. Bolsista CNPq do Programa de Iniciação Científica da UFMT. E-mail: aline_spanevello@hotmail.com ${ }^{2}$ Enfermeira. Doutora em Enfermagem. Docente da Faculdade de Enfermagem da UFMT. Diretora da FAEN/UFMT.

${ }^{3}$ Enfermeira. Mestranda do Programa de Pós Graduação - Mestrado em Enfermagem da FAEN/UFMT. Especialista em Auditoria de serviços de saúde. 
assistência de qualidade, uma vez que, por vezes, as Unidades Básicas de Saúde (UBS) não estão aptas a receber seus usuários, por diversas razões, seja em função de sua estrutura física não possibilitar seu funcionamento de maneira adequada, pela não existência de equipamentos e materiais de consumo necessários, pela falta de trabalhadores devidamente capacitados ou até mesmo pela gestão de serviço não ser implementada de maneira a atender às necessidades em saúde da população.

Segundo o MS, para resolver cerca de $85 \%$ dos problemas de saúde da comunidade faz-se necessário dispor de recursos estruturais e equipamentos compatíveis que possibilitem a ação dos profissionais de saúde ${ }^{(5)}$.

A persistência de problemas históricos na infraestrutura dos serviços de saúde reforça a disseminação de questionamentos sobre a efetividade da APS, relacionando-a, em alguma medida, às práticas profissionais e seus resultados ${ }^{(6)}$.

Frente ao exposto, neste estudo objetivou-se caracterizar a organização estrutural das unidades de ESF, do Município de Cuiabá-MT, para efetivação da assistência à prevenção dos cânceres de colo do útero e de mama, com vistas a melhor conhecer a realidade onde esta assistência se concretiza.

\section{METODOLOGIA}

Trata-se de um estudo descritivo e exploratório de abordagem quantitativa e delineamento transversal, realizado no município de Cuiabá, capital do estado de Mato Grosso - Brasil.

O município é constituído por uma rede básica composta de 85 UBS, sendo 22 Centros de Saúde e 63 USF, 10 clínicas odontológicas e uma unidade móvel que atende a 22 comunidades rurais ${ }^{(7)}$. Este estudo foi realizado com os profissionais de enfermagem responsáveis por 61 USF, uma vez que os enfermeiros de duas unidades estavam em período de férias, no período de março a maio de 2012.

Os dados foram coletados através do preenchimento de formulário pré-definido que foi aplicado às enfermeiras das USF no município de Cuiabá, sendo construído um bando de dados com utilização do software Epi Info 3.5.

Os dados levantados foram analisados através de análise estatística descritiva simples para implementação da caracterização das unidades estudadas, com uso do software Epi Info 3.5, e sua discussão foi implementada com base na literatura nacional sobre a temática.

O projeto matricial foi submetido ao Comitê de Ética em Pesquisa do Hospital Universitário Júlio Muller, com aprovação final № 090/CEP-HUJM/2011, atendendo ao previsto na Resolução n. ${ }^{\circ}$ 196/96 do Conselho Nacional de Saúde que normatiza o desenvolvimento de pesquisas que envolvam seres humanos.

\section{RESULTADOS}

Para caracterizar as unidades informamo-nos sobre a quantidade de pessoas e mulheres cadastradas sob responsabilidade da unidade. No entanto, das 61 unidades, 4 não souberam informar o número de pessoas cadastradas, o que corresponde a um total de 225.832 usuários para as 57 unidades. E ainda das 61, 12 também não souberam informar a quantidade de mulheres cadastradas, portanto, nas 49 unidades é cadastrado um total de 97.565 mulheres usuárias dos serviços.

A composição da Equipe de Saúde das USF estudadas, representada no Gráfico 1, nos revelou uma informação importante quanto à classe médica, pois 3, 3\% delas não possuíam nenhum profissional médico.

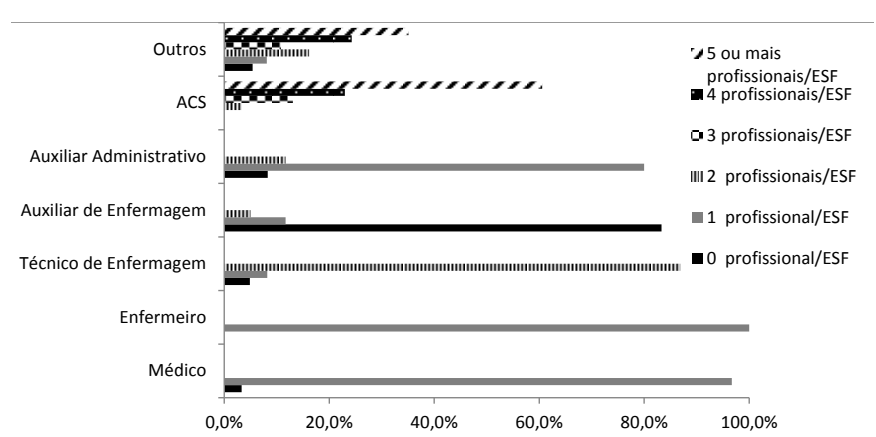

Grafico1 - Composição da equipe profissional das Unidades de Saúde da Família. Cuiabá/MT, 2012.

Em relação à equipe de enfermagem, em 100\% das unidades tinham minimamente um enfermeiro, sendo este o responsável pela equipe. No que diz respeito, aos profissionais de nível médio dessa classe, sendo eles os técnicos de enfermagem e os auxiliares, observamos que em $86,9 \%$ das USF havia dois técnicos e em 83 , 3\% deles não possuía nenhum auxiliar de enfermagem.

A quantidade de Agentes Comunitários de Saúde (ACS) variou de 2 a 11 profissionais, sendo que este número muda de acordo com a população que a unidade abrange.

No que diz respeito ao prontuário do usuário, perguntamos a todos os enfermeiros responsáveis pelas unidades quem utiliza e efetua registros no mesmo, desta forma, na totalidade das unidades pesquisadas tanto o médico, quanto o enfermeiro utilizam o prontuário do usuário para registros. Os dados mostraram que apenas $98,4 \%$ dos técnicos/auxiliares de enfermagem e $83,6 \%$ dos ACS, utilizam o prontuário efetuando registro.

Quanto aos resultados referentes à estrutura física das USF observa-se no Gráfico 2 que em 85, 2\% das unidades não havia sala de acolhimento, e em $96,7 \%$ delas havia uma sala de recepção. A existência de pelo menos um consultório de enfermagem e um consultório médico para atendimento foi identificado em todas as unidades (100\%). O expurgo foi encontrado em apenas $72,1 \%$ das unidades. $85,2 \%$ das unidades dispunha de sala específica para esterilização de material dessas unidades, no entanto 3, 8\% delas não estavam em funcionamento. Apenas 26, 2\% das unidades dispunha de abrigo para resíduos sólidos. A existência de uma sala para coleta de exames estava disponível em 90, 2\% das unidades.

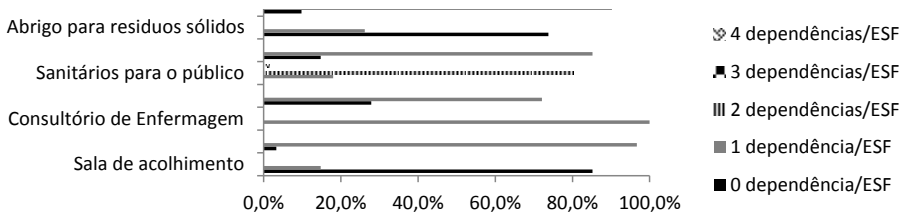

Gráfico 2 - Dependências das Unidades de Saúde da Família. Cuiabá/MT,2012.

Em relação à privacidade visual dos consultórios de enfermagem e médico encontramos que em $95,1 \%$ das unidades há privacidade em ambos os consultórios, sendo privacidade entendida como o usuário dentro de um desses consultórios fique a vontade por não estar sendo, ou mesmo sentir-se visto por ninguém fora dele. Em relação à existência de banheiro nos consultórios encontramos que em $86,9 \%$ dos consultórios de enfermagem havia banheiro, porém em $90,2 \%$ dos consultórios médico não havia banheiro. 


\section{DISCUSSÃO}

A falta de ACS/número reduzido detectado em algumas USF e o relato dos profissionais sobre as áreas descobertas pela falta destes profissionais leva-nos a refletir sobre sua importância na equipe. O ACS é o profissional que desempenha atividades juntamente com a comunidade, no qual objetiva, com estas, a prevenção de doenças e agravos por meio das visitas domiciliares e educação em saúde. É este profissional que possibilita que as necessidades da população cheguem até a Unidade de Saúde, exercendo, desta forma, a função de mediador entre a população e o serviço de saúde. Portanto, seu trabalho é de suma importância na efetivação da Estratégia Saúde da Família e consolidação do conceito ampliado de saúde $^{(8)}$. Desta forma, a falta desse profissional nas unidades conforma-se como importante empecilho para concretização dos trabalhos propostos para a prevenção dos cânceres de mama e colo do útero.

Em relação à estrutura física das USF, nota-se a falta de algumas dependências importantes para a execução do trabalho do enfermeiro, como sala de esterilização de material, abrigo para resíduos sólidos, dentre outros. A estrutura física influencia diretamente nas práticas dos enfermeiros, sendo essas práticas, portanto, influenciadas pelo sistema de saúde e suas limitações, gerando aos profissionais uma dificuldade na qualidade de suas práticas ${ }^{(9)}$.

Estudo realizado em Cuiabá-MT, no ano de 2010, em 18 UBS, sobre a influência da infraestrutura de centros de saúde nas práticas profissionais de enfermeiros demonstrou que menos da metade dos ambientes recomendados pelo Ministério da Saúde para a realização da assistência em APS estavam disponíveis nas UBS estudadas, o que representa um déficit no cumprimento dos critérios estabelecidos na normatização de ambientes ${ }^{(9)}$.

A gestão e a disposição inadequada dos resíduos sólidos, por sua vez, causam impactos socioambientais, tais como a degradação do solo, comprometimento dos corpos d'água e mananciais, intensificação de enchentes, contribuição para poluição do ar, proliferação de vetores de importância sanitária nos centros urbanos e catação em condições insalubres nas ruas e nas áreas de disposição final ${ }^{(10)}$. O gerenciamento adequado dos resíduos sólidos pode reduzir significativamente os impactos ao ambiente e à saúde. Portanto, a falta de um local adequado para depositar resíduos sólidos nas unidades, gera problemas sérios à população.

O acolhimento é uma ação considerada fundamental na APS, sendo que a inexistência de uma sala específica para o acolhimento do usuário nas unidades estudadas configura-se um problema. O acolhimento fortalece o PSF, uma vez possibilita ao usuário uma maior motivação para procurar o serviço e uma maior confiança na resolução de seus problemas, ${ }^{(11)}$ fortalecendo dessa forma o cuidado e permitindo uma maior adesão terapêutica e inclusão social.

\section{CONCLUSÃO}

A APS desempenha papel fundamental no rastreamento dos cânceres de colo de útero e de mama, e para que isso ocorra da maneira eficiente é necessária a qualificação e responsabilização dos profissionais envolvidos neste processo, o que requer a existência de um número suficiente de profissionais em cada USF.

O profissional de enfermagem, por sua vez, sendo um dos responsáveis pela USF, deve definir diagnosticar as necessidades em saúde da população adstrita e elaborar um plano de trabalho com vistas a atender essas necessidades. Entretanto, apesar de haver uma definição ministerial das funções/atribuições do enfermeiro que atua na ESF, com a realização deste estudo pudemos identificar que, os enfermeiros deixam de realizar algumas ações, gerando prejuízos à população local.

A falta de ACS nas unidades visitadas é um fator preocupante, uma vez que são estes profissionais os responsáveis por captar usuários na população, considerando que eles têm grande potencial para a detecção dos problemas que acometem a comunidade onde vivem e atuam. Sua proximidade com a comunidade permite o estabelecimento de vínculo, favorecendo a criação de uma ponte entre o serviço de saúde e o usuário, de maneira a identificar problemas de saúde e tentar alcançar soluções junto ao serviço de forma rápida e apropriada.

Para além, este estudo possibilitou a constatação da necessidade de melhorara estrutura física de várias unidades básicas de saúde, com vistas a qualificar a assistência a mulheres que procuram o serviço de saúde para a prevenção de cânceres de colo de útero e de mama.

Frente ao exposto fica evidente que, a assistência prestada a mulheres para prevenção do câncer de mama e de colo de útero, na Atenção Primária à Saúde no município de Cuiabá, ainda apresenta fragilidades que precisam ser revistas, considerando que esses tipos de cânceres apesar de apresentarem alta incidência no município, se identificados precocemente apresentam grande potencial de cura, sendo para tanto, fundamental que o serviço ofereça a população cuidado de qualidade 


\section{Referências}

1. Ministério da Saúde (BR). Instituto Nacional de Câncer (INCA). Estimativas 2010: incidência de câncer no Brasil. http://www. inca.gov.br/estimativa/2010/ (15 de novembro de 2012, data de acesso).

2. Ministério da Saúde (BR). Secretaria de Atenção à Saúde. Instituto Nacional do Câncer (INCA).http://www2.inca.gov.br/ wps/wcm/connect/inca/portal/home(12 de outubro de 2012, data de acesso)

3. Brasil. Ministério da Saúde. Secretaria de Atenção à Saúde. Departamento de Atenção Básica. Controle dos cânceres do colo do útero e da mama / Ministério da Saúde, Secretaria de Atenção à Saúde, Departamento de Atenção Básica. - 2. ed. - Brasília : Editora do Ministério da Saúde, 2013. 124 p.: il. (Cadernos de Atenção Básica, n. 13)

4. Brasil. Ministério da Saúde. Departamento de Atenção Básica - DAB. Atenção Básica e a Saúde da Família. http://dab.saude. gov.br/atencaobasica.php\#saudedafamilia (20 de novembro de 2012, data de acesso).

5. Brasil. Ministério da Saúde. Secretaria de Atenção à Saúde. Departamento de Atenção Básica. Manual de estrutura física das unidades básicas de saúde: saúde da família / Ministério da Saúde, Secretaria de Atenção à Saúde, Departamento de Atenção Básica - 2. ed. - Brasília : Ministério da Saúde, 2008. 52 p.: il. color - (Série A. Normas e Manuais Técnicos).

6. Facchini LA, Piccini RX, Tomasi E, Thumé E, Silveira DS, Siqueira FV, Rodrigues MA.Desempenho do PSF no Sul e no Nordeste do Brasil: avaliação institucional e epidemiológica da Atenção Básica à Saúde. Ciênc. saúde coletiva, Rio de Janeiro,
2006; 11 (3). http://www.scielosp.org/scielo.php?script=sci_ arttext\&pid=S1413-81232006000300015\&lng=pt\&nrm=isso ( $18 \mathrm{de}$ maio de 2012, data de acesso).

7. Cuiabá. Secretaria Municipal de Saúde: Plano Municipal de Saúde 2010-2013. SUS/CUIABÁ, Cuiabá 2010.

8. Costa S de M, Araújo FF, Martins LV, Nobre LR, Araújo FM, Rodrigues CAQ. Agente Comunitário de Saúde: elemento nuclear das ações em saúde. Rev Ciênc. saúde coletiva. 2013; 18(7).

Disponível em http://www.scielosp.org/scielo.php?script=sci_artte xt\&pid=S1413-81232013001500030 (19 de janeiro de 2013, data de acesso).

9. Pedrosa ICF, Corrêa ACP, Mandú ENT. Influências da Infraestrutura de Centros de Saúde nas Práticas Profissionais: Percepções de Enfermeiros. Rev. Cienc. Cuid.Saúde, 2011; 10(1);58-65. Disponível em: http://periodicos.uem.br/ojs/index.php/CiencCuidSaude/ article/viewFile/13288/pdf(17 de novembro de 2012, data de acesso)

10. Besen GR, et al. Resíduos sólidos: vulnerabilidade e perspectivas. In: Saldiva P. et al. Meio ambiente e saúde: o desafio das metrópolis. São Paulo 2010.

11. Costa C de O, Costa CFS, Vaaghettic HH. Acolhimento no processo de trabalho da enfermagem: estratégia para adesão ao controle do câncer do colo uterino. Rev. Baiana de Saúde Pública. 2010 jul-set; 34(3);706-717. Disponível em http://inseer.ibict.br/ rbsp/index.php/rbsp/article/viewFile/67/66 (20 de janeiro de 2013, data de acesso) 